\title{
Estudio del coeficiente emocional en estudiantes de medicina
}

\section{The emotional coefficient in medicine students}

\author{
Dexy Prieto-Rincón ${ }^{1}$, José Inciarte-Mundo ${ }^{2}$, Ciro Rincón-Prieto ${ }^{2}$ y Ernesto Bonilla ${ }^{1,3}$
}

The Emotional Coefficient (EQ) of 93 third year medical students was determined by means of the Emotional Coefficient test of Boeck and Martin. The test gives quantitative results ranging from 0 (minimum) to 160 (maximum). The sample studied included 28 male and 65 female students, The EQ mean of the whole sample was 77.68 points qualifying them as emotionally competent people with a average emotional intelligence. The distribution of the EQ was as follows: 21 (22.59\%) students in the range of 0-60 points; 32 (34.40\%) with 61-80 points and 40 (43.01\%) with 81-160 points. We believe that efforts to improve the EQ would be beneficial to the students, as well as to the patients that will be under their care when they graduate, and suggest that EQ be determined in all medical students in order to evaluate and improve their personal and professional growth.

Key words: Emotional Intelligence, medical students, Emotional Coefficient. Rev Chil Neuro-Psiquiat 2008; 46 (1): 10-15

\section{Introducción}

$\mathrm{L}$ a palabra inteligencia etimológicamente deriva del latín "intellegere", que significa "recolectar de entre", lo que le atribuye una connotación relacionada al discernimiento o toma de decisiones $^{1}$. La prueba de inteligencia más antigua fue creada por Alfred Binet y Theodore Simon en 1905, en un primer intento de objetivar esta función².
En 1916, Lewis Terman introdujo el término "Coeficiente Intelectual" (CI), buscando una correlación con el "éxito" estudiantil y laboral ${ }^{2}$. Weschler (1944) ideó la prueba de inteligencia más usada actualmente, que incluye subpruebas verbales (verbal, atención y concentración) y subpruebas manuales (coordinación visuomotora y organización visual). En su libro "La medición de la inteligencia en los adultos" escribe: "Inteligencia es la facultad compuesta o global del indi-

Recibido: 10 de mayo de 2007

Aceptado: 31 de marzo de 2008

Centro de Investigaciones Biomédicas (IVIC-Zulia), Maracaibo, Venezuela.

Escuela de Medicina. Universidad del Zulia, Maracaibo, Venezuela.

Instituto de Investigaciones Clínicas “Dr. Américo Negrette”. Facultad de Medicina, Universidad del Zulia. Maracaibo, Venezuela. 
viduo de actuar adecuadamente, pensar razonablemente y relacionarse efectivamente con su mundo circundante"3. En el año 1990, Peter Salovey y John Mayer propusieron por primera vez el modelo de "Inteligencia Emocional" y describieron los principales aspectos que la conforman, siendo estos: el conocimiento y manejo de las propias emociones, la automotivación, el reconocimiento de las emociones de los demás y el manejo de las relaciones interpersonales ${ }^{4}$.

De esta forma, podemos deducir que la inteligencia es un concepto muy amplio, que implica la capacidad del individuo para llevar a término exitosamente una empresa, e incluye la Inteligencia Cognitiva (IC) y la Inteligencia Emocional (IE). La IC se compone de facultades como la atención, la memoria, el lenguaje y la abstracción, mientras que la IE incluye elementos sociales y emocionales. Estos últimos no son medibles con las pruebas de inteligencia habituales ${ }^{5}$.

El desarrollo de la IE es crucial para alcanzar el éxito en la vida, incluso tanto o más que la IC $y$, a diferencia de ésta, tendría una mayor carga ambiental que genética. Es por esto que, pese a su reciente descripción, la IE se ha convertido en uno de los temas más debatidos en psicología y más estudiados en neurología conductual, amenazando con crear una verdadera revolución en los métodos de enseñanza tradicionales, los que están enfocados principalmente al desarrollo de la IC.

Podemos entonces definir el Coeficiente Emocional (CE) como el resultado de la medición de la capacidad del individuo de sentir, entender y controlar los estados anímicos propios y de las personas que le rodean. Aquellas personas que tengan un CE elevado serán socialmente equilibradas, alegres, decididas, responsables y se sentirán cómodas consigo mismo y capaces de relacionarse con el mundo que les rodea ${ }^{5}$.

El presente trabajo se realizó con el fin de determinar el Coeficiente Emocional (CE) de los estudiantes del tercer año de Medicina en la Universidad del Zulia (Maracaibo, Venezuela) para establecer la distribución del CE en esta población estudiantil y conocer si existe alguna relación entre el sexo y el CE.

\section{Material y Métodos}

Se realizó un estudio cuantitativo para determinar el CE de los estudiantes de tercer año de Medicina de La Universidad del Zulia, todos cursantes de la materia Psicología Médica. Se utilizó la prueba del Coeficiente Emocional de Boeck y Martin $^{6}$, para cuantificar el coeficiente emocional de 93 estudiantes de una población total de 120 personas.

Para la estimación del tamaño de la muestra se siguió el procedimiento descrito por Motulsky ${ }^{7}$. Para la selección de los estudiantes que conformaron la muestra se utilizó la técnica de azar simple, entre el grupo de estudiantes cursantes de Psicología Médica en los tres años que abarcó el estudio. La población estudiada estuvo constituida por 28 estudiantes del sexo masculino $(30,11 \%)$ y 65 del sexo femenino $(69,89 \%)$. Los perfiles de sexo y edad de los sujetos fueron comparables a los de la población general de 120 estudiantes.

La prueba del coeficiente emocional de Boeck y Martin ${ }^{6}$, es una forma abreviada de la prueba de Inteligencia Emocional utilizado por Goleman ${ }^{5}$. Es una encuesta de ocho preguntas cerradas en donde se le plantean al entrevistado situaciones hipotéticas y cuatro opciones cerradas de cómo actuaría frente a dichas situaciones, que al ser seleccionadas pueden producir una calificación de 0, 5 ó 20 puntos. Toda la encuesta es llenada por el entrevistado, dejándose criterio para el anonimato, respetando así la privacidad, pero destacándose al inicio datos importantes para las variables utilizadas en la investigación como: sexo, edad y escolaridad ${ }^{5,6}$. Los estudiantes anotaron sus resultados en un formato precodificado preparado para la mejor tabulación de los resultados logrados, asegurando la calidad de la base de datos obtenida con esta investigación.

Se obtuvieron los permisos reglamentarios del Hospital Psiquiátrico de Maracaibo y de la Cátedra de Psicología Médica que estuvo informada de todos los procedimientos que se iban a seguir así como también del propósito de la investigación. 
Tabla 1. Distribución del coeficiente emocional de los estudiantes del tercer año de Medicina de la Universidad del Zulia

\begin{tabular}{lcc}
\hline $\begin{array}{l}\text { Coeficiente } \\
\text { emocional }\end{array}$ & $\begin{array}{c}\text { No de } \\
\text { Estudiantes }\end{array}$ & $\%$ \\
\hline 0-60 puntos & 21 & 22,59 \\
61-80 puntos & 32 & 34,40 \\
$81-160$ puntos & 40 & 43,01 \\
Total & 93 & 100 \\
\hline
\end{tabular}

\section{Resultados}

La puntuación total media del CE de toda la muestra fue 77,68 puntos. La distribución de la muestra total estudiada según el CE obtenido fue la siguiente: $0-60$ puntos en 21 personas, que representaron un 22, 59\% de la muestra total; 6180 puntos en 32 personas, que representaron un $34,40 \%$ de la muestra total; $81-160$ en 40 personas, $43,01 \%$ de la muestra consultada (Tabla 1 ). En la muestra correspondiente al sexo femenino se obtuvieron los siguientes datos: 12 estudiantes $(18,46 \%)$ lograron una puntuación entre 0 y 60 puntos; 26 estudiantes (40\%) obtuvieron entre 61-80 puntos y el tercer grupo, el más numeroso (27 personas, 41,54\%) tuvo un elevado grado de IE. La puntuación promedio entre la población femenina estudiada fue de 78,92 puntos calificándolos como competentes emocionales dentro de los límites normales (Tabla 2). Dentro de la población masculina estudiada 9 estudiantes $(32,14 \%)$ obtuvieron una puntuación de 0-60 puntos necesitando ayuda profesional, 6 estudiantes $(21,43 \%)$ obtuvieron una puntuación de 6180 puntos calificándolos como competentes emocionales, y 13 estudiantes $(46,43 \%)$ presentaron una puntuación mayor a 80 puntos lo cual representa un elevado grado de inteligencia emocional. La puntuación promedio para el sexo masculino fue de 77,68 puntos calificándolos como competentes emocionales dentro de los límites normales (Tabla 3). La edad de los estudiantes del sexo femenino fue de $20 \pm 1,2$ años y la de los
Tabla 2. Distribución de la población femenina según coeficiente emocional

\begin{tabular}{lcc}
\hline $\begin{array}{l}\text { Coeficiente } \\
\text { emocional }\end{array}$ & $\begin{array}{c}\text { No de } \\
\text { Estudiantes }\end{array}$ & $\%$ \\
\hline 0-60 puntos & 12 & 18,46 \\
61-80 puntos & 26 & 40,00 \\
$81-160$ puntos & 27 & 41,54 \\
Total & 65 & 100 \\
\hline
\end{tabular}

Tabla 3. Distribución de la población masculina según coeficiente emocional

\begin{tabular}{lcc}
\hline $\begin{array}{l}\text { Coeficiente } \\
\text { emocional }\end{array}$ & $\begin{array}{c}\text { No de } \\
\text { Estudiantes }\end{array}$ & $\%$ \\
\hline $0-60$ puntos & 9 & 32,14 \\
61-80 puntos & 6 & 21,43 \\
$81-160$ puntos & 13 & 46,43 \\
Total & 28 & 100 \\
\hline
\end{tabular}

del sexo masculino fue de $20 \pm 0,9$. No se detectó ninguna diferencia en la edad entre ambos sexos.

\section{Discusión}

La muestra estudiada calificó como formada por competentes emocionales, con inteligencia emocional promedio. La muestra fue extraída cuando los alumnos apenas comienzan sus prácticas en las instituciones hospitalarias; quizás no han tenido mucho roce con pacientes y no han podido desarrollar o probar, con situaciones vividas por ellos mismos, su inteligencia emocional. Sería interesante realizar un estudio longitudinal con estudiantes, desde el comienzo de su carrera hasta su culminación, cuando estén en capacidad de diagnosticar y tratar pacientes, para medir cuánto han desarrollado su coeficiente intelectual y cómo lo ponen en práctica en la resolución de problemas básicos de la profesión. 
En ambos sexos el mayor grupo de personas encuestadas entró en el tercer grupo de coeficientes emocionales, con una inteligencia superior al promedio. Las diferencias de edad y sexo no fueron significativas y por lo tanto, no constituyeron una variable determinante en el desarrollo del CE de los sujetos estudiados.

Las habilidades y destrezas emocionales son fácilmente adquiridas. A diferencia del CI que es difícil de desarrollar, el CE es potencialmente desarrollable en cualquier persona, razón por la cual se debe incentivar a los estudiantes de las ciencias de la salud, a desarrollar dichas habilidades e informarle de las ventajas y privilegios de estar en contacto con sus emociones y ser asertivos al hacerlo ${ }^{8}$.

Se han descrito cientos de casos en donde la IE se aplica a problemas de salud: por ejemplo, en algunos hospitales han desarrollado un instructivo programa prequirúrgico para pacientes, que los ayuda a aliviar los miedos y a abordar las molestias enseñándoles técnicas de relajación, respondiendo a sus preocupaciones e informándoles varios días antes de la operación exactamente qué les ocurrirá durante la recuperación. El resultado es que los pacientes se recuperan de la cirugía dos o tres días más pronto9. Es evidente que existe una estrecha relación entre el estado de ánimo y la evolución de la enfermedad, y el saber manejar situaciones con gran carga afectiva podría representar la diferencia entre la vida y la muerte.

Tal vez el testimonio más revelador de la capacidad curativa de los vínculos emocionales es un estudio sueco publicado en 1993. Todos los hombres que vivían en la ciudad sueca de Göteborg y que habían nacido en 1933 fueron sometidos a un examen médico gratuito; siete años más tarde, los 752 hombres que se habían presentado al examen fueron convocados nuevamente. De éstos, 41 habían muerto en los años transcurridos ${ }^{10}$. Los hombres que originalmente habían informado que se encontraban sometidos a una intensa tensión emocional tenían un índice de mortalidad tres veces mayor que aquellos que reportaron que su vida era serena y plácida. La aflicción emocional se debía a acontecimientos tales como un grave problema financiero, sentirse inseguro en el trabajo o quedar despedido del empleo, ser objeto de una acción legal o divorciarse. Haber tenido tres o más de estos problemas en el curso del año anterior al examen fue un pronosticador más claro de muerte, dentro de los siete años siguientes, de lo que fueron indicadores médicos tales como la elevada presión sanguínea, las altas concentraciones de triglicéridos en la sangre, o los niveles elevados de colesterol sérico. Sin embargo, entre los hombres que dijeron que tenían una red confiable de intimidad -una esposa, amigos íntimos- no existió ningún tipo de relación entre los niveles elevados de estrés y el índice de mortalidad. Tener a quién recurrir y con quién hablar, alguien que podía ofrecer consuelo, ayuda y sugerencias, los protegía del mortal impacto de los rigores y los traumas de la vida.

Se han utilizado pruebas proyectivas que relacionan efectivamente variables psicológicas con la presencia de un tumor maligno ${ }^{11}$. Las pacientes con cáncer de mama por ejemplo, que presentan índices de sobrevida más prolongados son aquéllas que adoptan actitudes más combativas, con 'espíritu de lucha', más que confiadas y complacientes con la enfermedad ${ }^{12}$. Adicionalmente, las personas con baja estima tienen una probabilidad superior al promedio de contraer enfermedades cardíacas y pulmonares. También es más probable que se vean afectadas por enfermedades autoinmunes y sufran el avance vertiginoso de los tumores malignos. Las pruebas muestran que los individuos con excesiva confianza en sí mismos tienen una alta tolerancia al dolor y al impacto quirúrgico, presentan un riesgo menor al promedio de sufrir de artritis y alergias y poseen una alta resistencia a la mayoría de las infecciones $^{13}$.

En otro estudio, 122 hombres que tuvieron su primer ataque cardíaco fueron evaluados para determinar su grado de optimismo o pesimismo. Ocho años más tarde, de los 25 hombres más pesimistas, 21 habían muerto; de los 25 más optimistas, sólo 6 habían muerto. Su estado mental 
demostró ser un mejor pronosticador de la supervivencia que cualquier otro factor de riesgo, incluido el grado de daño sufrido por el corazón en el primer ataque, bloqueo de arterias, nivel de colesterol o presión sanguínea ${ }^{14}$. Así mismo, en pacientes con insuficiencia renal crónica que estaban recibiendo diálisis, aquéllos a los que se le diagnosticó depresión grave tenían más posibilidades de morir dentro de los dos años posteriores; la depresión fue un pronosticador más decisivo de muerte que ninguna otra señal médica ${ }^{15}$.

Es importante señalar que los hospitales, vistos como organizaciones, también necesitan de- sarrollar la inteligencia emocional. Una organización hospitalaria (pacientes, médicos, empleados, obreros) emocionalmente inteligente genera posibilidades infinitas de éxito y resulta un instrumento eficaz para resolver una situación delicada con un paciente o con un compañero de trabajo. De allí la necesidad de identificar y potenciar la IE de todos los integrantes del equipo de salud, especialmente de los estudiantes de las ciencias de la salud y del personal médico y de enfermería, con miras a obtener mejores resultados en los índices de evolución y curación de los pacientes bajo su cuidado.

\section{Resumen}

Se cuantificó el Coeficiente Emocional (CE) de 93 estudiantes del tercer año de medicina utilizando la prueba del Coeficiente Emocional de Boeck y Martin. La prueba da resultados en rangos desde 0 (minimo) hasta 160 (máximo). La población estudiada estuvo constituida por 28 estudiantes del sexo masculino y 65 del sexo femenino. La puntuación media del CE de toda la muestra fue 77,68 puntos, calificándola como constituida por competentes emocionales, con inteligencia emocional promedio. La distribución del CE en la muestra estudiada fue la siguiente: 21 (22,59\%) estudiantes en el rango de 0-60 puntos; 32 (34,40\%) con 61-80 puntos y 40 (43,01\%) con 81-160 puntos. Creemos que todos los esfuerzos que se realicen para mejorar este CE serían beneficiosos tanto para los estudiantes como para los pacientes que estarán bajo su cuidado cuando terminen sus estudios, razón por la cual recomendamos que se determine el CE en todos los estudiantes de medicina con el fin de evaluar y mejorar su evolución personal y profesional.

Palabras clave: Inteligencia emocional, coeficiente emocional, estudiantes de medicina.

\section{Referencias}

1. Wolff W. La inteligencia, en Introducción a la Psicología. $1^{\mathrm{TM}}$ d. México. Fondo de Cultura Económica, 1953.

2. Adams R, Victor M. The Limbic Lobes and the Neurology of Emotion, en Principles of Neurology. $5^{\mathrm{TM}}$ ed. N.York. McGraw Hill, 1993.

3. Weschler D. The Measurement and Appraisal of Adult Intelligence (4th Ed). Baltimore, Williams and
Wilkins, 1958.

4. Salovey P, Mayer J D. Emotional Intelligence. Imagination. Cognition and Personality 1990; 9: 185-211.

5. Goleman D. La Inteligencia Emocional. B. Aires. Javier Vergara Editor S.A., 1996.

6. Boeck K, Martin D. Inteligencia Emocional. Editorial Edaf. Barcelona. 1997.

7. Motulsky H. Intuitive Biostatistics. New York, Oxford University Press 1995; p 195-204. 
8. Weisinger H. La inteligencia emocional en el trabajo. Ed. Javier Vergara, Buenos Aires, 1998.

9. LeShan L. An emotional life-history pattern associated with neoplastic disease. Ann N Y Acad Sci 1966; 125 : 780-93.

10. Peterson C, Seligman P, Vaillant G. Pessimistic explanatory style is a risk factor for physical illness: a thirty-five-year longitudinal study, J of Personality and Social Psychology, 1988.

11. Thomas C B. Precursors of premature disease and death : The predictive potential of habits and family attitudes. Ann Intern Med 1976; 85: 653-8.

12. Derogatis L. Breast and gynaecologic cancers: Their unique impact on bogy image and sexual identity in women', en J. Vaeth, R. Blomber y L. Adler, Frontiers of Radiation Therapy and Oncology, vol. 14, Body Image Self-esteem and Sexuality in Cancer Patients, Basel Switzerland, Karger, pp 1-11.

13. Engel B T. Psychosomatic Medicine, Behavioral Medicine, Just Plain Medicine. Psychosom Med 1986; 48: 466-79.

14. Peterson C. Learned helpnessness: a theory for the age of personal control, Oxford University Press, 1993.

15. Burton H J, Kline S A, Lindsay R M, Heidenheim A P. The Relationship of Depression to Survival in Chronic Renal Failure', Psychosom Med 1986; 48: 261-9.

Correspondencia:

Dra. Dexy Prieto de Rincón

Centro de Investigaciones Biomédicas

(IVIC-Zulia). Apartado Postal 121.

Maracaibo, Venezuela. Tlf. 261-7916707

E-Mail: dexyprieto@hotmail.com 